\title{
CONTROLE FUZZY-PID DE UM SISTEMA DE BOMBEIO CENTRIFUGO SUBMERSO
}

\author{
LEONARDO DA F. SOUZA, LUIZ H. S. TORRES, LEIZER SCHNITMAN
}

\author{
Centro de Capacitação Tecnológica em Automação Industrial (CTAI), Programa de Pós-Graduação em \\ Mecatrônica da Universidade Federal da Bahia, Rua Aristides Novis, $n^{\circ} 02$, Escola Politécnica, $2^{\circ}$ andar, \\ 40.210-630, Salvador, Bahia, Brasil \\ E-mails:leo_fsouza@oi.com.br, luizhenrique@ieee.org, leizer@ufba.br
}

\begin{abstract}
The Electrical Submersible Pump (ESP) is one of the methods used for artificial lift of oil. Thus, this paper proposes the use of Fuzzy-PID control technique to control the level of dynamic fluid in the annular well, even in the presence of uncertainty and dynamics not modeled. This nonlinear controller is able to track and monitor a system, also non-linear. The Laboratório de Elevação Artificial (LEA), da Escola Politécnica da Universidade Federal da Bahia, has a plant ESP with a oil well $32 \mathrm{~m}$ instrumented, in a laboratory environment that favorece the development of studies and research, among others, in the control area. The aim of this study is to control the level of viscous fluid (oil) in the annular well. The results obtained with the method of fuzzy PID control in the simulated environment shows that there is stability in the control signal and response of the controlled plant.
\end{abstract}

Keywords: Fuzzy-PID Control, Hybrid Control, Intelligent Control, ESP, Artificial Lift Oil.

\begin{abstract}
Resumo: O Bombeio Centrifugo Submerso (BCS) é um dos métodos aplicados para a elevação artificial de petróleo. Assim, este artigo propõe o uso de técnica de controle Fuzzy-PID para controlar o nível dinâmico de fluido no anular do poço, mesmo na presença de incertezas e dinâmicas não modeladas. Este controlador não-linear é capaz de controlar um sistema, também não-linear. O Laboratório de Elevação Artificial (LEA), da Escola Politécnica da Universidade Federal da Bahia, dispõe de uma planta BCS com um poço de $32 \mathrm{~m}$ de altura completamente instrumentado, num ambiente experimental que favorece o desenvolvimento de estudos e pesquisas, entre outros, na área de controle. O objetivo deste estudo é controlar o nível de fluido viscoso (óleo) no anular do poco. Os resultados obtidos com o método de controle Fuzzy-PID em ambiente simulado mostram que há estabilidade no sinal de controle e resposta da planta controlada.
\end{abstract}

Palavras-chave: Controle Fuzzy-PID, Controle Híbrido, Controle Inteligente, BCS, Elevação Artificial de Petróleo.

\section{Introdução}

Tem crescido nos últimos anos, a utilização do método de elevação artificial de petróleo denominado de Bombeio Centrífugo Submerso (BCS). Segundo Ribeiro et al. (2005), a Petrobrás S.A. é pioneira no uso de bombas centrifugas submersas submarina em águas profundas, por meio de um teste do protótipo bem sucedido em um poço submarino, em 1994. Este tipo de bombeio consiste na transmissão de energia elétrica, por meio de cabo elétrico, para um motor de sub-superfície imerso no óleo, no fundo do poço. O motor tem seu eixo conectado a uma bomba centrifuga que incrementa pressão ao fluido, fazendo com que chegue até a superfície (Estevam, 2002; Rossi, 2008). O ambiente em que a bomba BCS é instalada é composto por fluido multifásico (água, óleo e gás), baixa pressão de reservatório e variação de temperatura.

O BCS trabalha com faixa larga de vazões volumétricas e é responsável pelas maiores quantidades de líquido bombeado por um único método de elevação artificial (Maitelli, 2010). Um ponto importante neste método de elevação é o conhecimento das curvas características da bomba para o correto dimensionamento e controle do processo de produção de petróleo. As curvas características representam a trajetória de desempenho de uma bomba BCS e sua faixa de operação recomendada pelo fabricante que em seus catálogos considera o fluido como sendo a água. Neste sentido é proposta deste artigo: a correção de viscosidade do fluido (Turzo et al., 2000 apud Estevam, 2008; Takács, 2009) e aplicação do controle Fuzzy-PID (Lemke, 1985). O controlador aqui aplicado visa controlar o nível do fluido no anular do poço, mesmo na presença de incertezas e dinâmicas não modeladas associadas a este processo de bombeio.

Este artigo está organizado na seguinte forma: a seção 2 apresenta o perfil de escoamento de fluido, leis de afinidades e cálculos de correção de viscosidade conforme o Hydraulic Institute-USA. Na seção 3 é descrito algumas não linearidades do sistema BCS e o método Fuzzy-PID. A seção 4 apresenta os resultados em ambiente simulado dos sinais de controle. E finalmente, a seção 5 traz as conclusões.

\section{Método de elevação de petróleo por BCS}

Existem diversos métodos de elevação artificial de petróleo cujo objetivo em comum é fornecer pressão ao fluido para sua elevação até a superfície. A escolha de um determinado método depende de vários critérios técnicos inerente ao reservatório e poço de produção. No caso específico do BCS, pode-se citar a limitação que o processo tem em relação à elevação de fluidos com grandes volumes de gás (traduzidas pelo RGO - razão gás-óleo), uma vez que a presença de bolhas de gás no fluido faz com 
que se perca eficiência no bombeamento. Em presenças de grandes volumes de gás pode-se chegar ao ponto de bloqueio da bomba e conseqüente parada da produção. A condição básica do método é que a bomba BCS esteja totalmente imersa no óleo para seu funcionamento.

\subsection{Escoamento monofásico}

Neste artigo considera apenas o escoamento de óleo no sistema BCS, denominado escoamento monofásico. Este escoamento é caracterizado por um alto fluxo de líquido incompressível e se houver gás, é uma pequena e desprezível fração na admissão da bomba, de forma que não afete o desempenho do processo (Verde, 2011). Neste sentido o LEA possui uma planta BCS reduzida com um poço artificial de $32 \mathrm{~m}$ de altura, totalmente instrumentado com sistema de supervisão, controle, coleta e registro dos dados. A referida planta, possui uma válvula no topo do processo de elevação, a qual pode limitar a passagem do fluido produzido pela elevação. Devido a isso, quando a válvula é parcialmente fechada, a pressão de descarga (saída da bomba) aumenta a ponto de possibilitar a realização de experimentos mais próximos da realidade, representando poços com profundidades maiores.

Para o presente estudo foram realizados experimentos práticos com o intuito de representar a curva do reservatório físico conhecida como índice de produtividade do reservatório (IPR). O IPR de um poço varia ao longo dos anos, mas é considerado constante durante um determinado período de produção (meses ou às vezes anos). Representa a capacidade de vazão volumétrica por diferencial de pressão fornecido pelo reservatório de produção. Outra grandeza importante é denominada de pressão estática do reservatório $P_{r}$, que cai ao longo de anos de produção mas é considerada constante por um determinado período. As condições técnicas do poço tais como: deposição de parafina, alteração de percentual de água e sedimentos, e formação de emulsão também podem modificar o comportamento dinâmico de um poço que, na proposta atual, podem ser considerados como parte das perturbações ou dinâmicas não modeladas. O IPR representa a capacidade de vazão volumétrica por diferencial de pressão fornecido pelo reservatório de produção.

A equação de Darcy, largamente utilizada na literatura, representa a curva $I P R$ de um escoamento monofásico, quando seu comportamento é linear. A Equação (1) mostra a conhecida lei de Darcy, onde $Q_{r}$ é a vazão volumétrica; $P_{r}$ é a pressão estática; $P_{w f}$ é a pressão dinâmica de fundo e o $I P R$, já mencionado, é o índice de produtividade do reservatório.

$$
Q_{r}=\operatorname{IPR}\left(P_{r}-P_{w f}\right)
$$

Na Fig(1), observa-se uma reta que representa a curva IPR do reservatório, e dois pontos $P_{1}$ e $P_{2}$ em que o sistema está operando com vazão volumétrica constante. Não está no SI, mas na unidade usualmente tratada nos campos de produção.

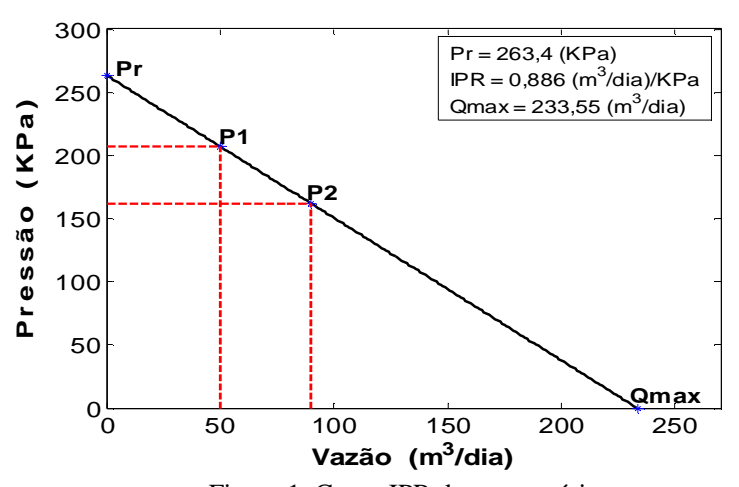

Figura 1. Curva IPR do reservatório.

Quando ocorre uma mudança no ponto de operação da bomba devido à utilização de um inversor de freqüência, também ocorre proporcionalmente uma modificação na vazão do fluido. Esta modificação provoca mudanças na curvas características que são previsíveis e podem ser determinadas por equações denominadas Leis de Afinidades. Estas leis relacionam a velocidade de rotação da bomba, $N$, com a vazão, $Q$, altura de elevação, $H$, e potência, Pot. Conforme mostrado na Equação (2).

$$
\frac{Q_{2}}{Q_{1}}=\frac{N_{2}}{N_{1}}=\sqrt{\frac{H_{2}}{H_{1}}}=\sqrt[3]{\frac{\text { Pot }_{2}}{\text { Pot }_{1}}}
$$

Onde se considera o subscrito 1 (um) referenciando, por exemplo, a condição inicial no ponto $P_{l}$ e o subscrito 2 (dois) à nova condição de operação $P_{2}$.

\subsection{Bombeio centrifugo submerso}

O BCS é composto por vários estágios mecânicos que servem para multiplicar a pressão incrementada ao fluido, correspondendo assim a uma faixa de valores de vazão e nível (elevação do fluido) em função da frequiência que aciona a bomba instalada no poço. O desempenho do BCS pode ser analisado por meio de curvas características que relacionam as grandezas: nível, potência e rendimento versus vazão. Entretanto, as curvas fornecidas pelos fabricantes por meio de catálogos consideram o fluido como sendo a água, por isso é necessário realizar uma correção de viscosidade para o fluido utilizado no sistema de bombeio.

Um método clássico para correção de viscosidade é proposto pelo Hydraulic Institute - USA, que utiliza dois diagramas, disponíveis na literatura, para se determinar os fatores de correção de vazão volumétrica, $C_{\mathrm{q}}$, altura de elevação, $C_{\mathrm{h}}$, e eficiência, $C_{\eta}$. Os fatores de correção da curva característica são utilizados na análise de desempenho da bomba, quando operando com fluido viscoso (Amaral, 2007; Takács, 2009). As Eq.(3), Eq.(4) e Eq.(5) apresentam a relação entre a vazão, $Q_{\text {óleo }}$, altura de elevação, $H_{\text {óleo }}$, e eficiência, $\eta_{\text {óleo }}$, do fluido viscoso. 
Com os fatores de correção: $C_{\mathrm{q}}, C_{\mathrm{h}}$ e $C_{\eta}$. Os termos: $Q_{\text {água }}, H_{\text {água }}$ e $\eta_{\text {água }}$ correspondem a vazão, altura de elevação e rendimento considerando o referencial água, respectivamente.

$$
\begin{gathered}
Q_{\text {óleo }}=C_{q} Q_{\text {água }} \\
H_{\text {óleo }}=C_{h} H_{\text {água }} \\
\eta_{\text {óleo }}=C_{\eta} \eta_{\text {água }}
\end{gathered}
$$

A correção de viscosidade elaborada pelo $\mathrm{Hi}$ draulic Institute - USA, envolve leitura visual por meio de diagramas, que podem gerar uma análise demorada e imprecisa na determinação dos fatores de correção de viscosidade. Neste sentido, Turzo et al., (2000) apud (Estevam, 2008; Takács, 2009), a fim de melhorar a análise das referidas correções, desenvolveram algumas funções numéricas para determinação dos fatores de viscosidade com base nos já mencionados gráficos do hidraulic Institute USA.

As equações desenvolvidas por Turzo são aplicadas neste artigo. As Eq.(6) e Eq.(7) representam os parâmetros de estimação: $y$ e $Q^{*}$. Estes parâmetros são utilizados na correção de viscosidade. A Eq.(6) utiliza a vazão volumétrica, $Q_{\text {bep }}$, e altura de elevação $H_{\text {bep }}$ da curva característica da bomba, fornecida nos catálogos com o ponto BEP (Best Efficiency Point). Já a Eq.(7) utiliza o parâmetro calculado, $y$, e a viscosidade cinemática do fluido, $v$.

$$
\begin{gathered}
y=-112,1374+6,6504 * \ln \left(H_{b e p}\right)+12,8429 * \ln \left(Q_{\text {bep }}\right) \\
Q^{*}=\exp \left(\frac{39,5276+26,5605 * \ln (v)-y}{51,6565}\right)
\end{gathered}
$$

O fator de correção da vazão volumétrica é determinado pela Eq.(8). E o fator de correção da eficiência da bomba centrifuga é calculado pela Eq.(9). Ambos os fatores são constantes para determinação de qualquer outro ponto de correção, quer seja vazão ou eficiência.

$$
\begin{gathered}
C_{Q}=1,0-4,0327 * 10^{-3} Q^{*}-1,724 * 10^{-4}\left(Q^{*}\right)^{2} \\
C_{\eta}=1,0-3,3075 * 10^{-2} Q^{*}+2,8875 * 10^{-4}\left(Q^{*}\right)^{2}
\end{gathered}
$$

A altura de elevação do líquido viscoso se baseia na determinação de quatro pontos distintos, que definem a trajetória da curva corrigida de elevação do fluido. Os quatro pontos correspondem aos seguintes percentuais considerando o fluido sendo óleo: Eq.(10) $60 \%$ do BEP, $C_{\mathrm{H} 0,6}$, Eq.(11) $80 \%$ do BEP, $C_{\mathrm{H} 0,8}$, Eq.(12) $100 \%$ do BEP, $C_{\mathrm{H} 1,0}$, e Eq.(13) $120 \%$ do BEP, $C_{\mathrm{H} 1,2}$.

$$
\begin{aligned}
& C_{H 0,6}=1,0-3,68 * 10^{-3} Q^{*}-4,36 * 10^{-5}\left(Q^{*}\right)^{2} \\
& C_{H 0,8}=1,0-4,4723 * 10^{-3} Q^{*}-4,18 * 10^{-5}\left(Q^{*}\right)^{2} \\
& C_{H 1,0}=1,0-7,00763^{*} 10^{-3} Q^{*}-1,41 * 10^{-5}\left(Q^{*}\right)^{2}
\end{aligned}
$$

$$
C_{H 1,2}=1,0-9,01 * 10^{-3} Q^{*}+1,31 * 10^{-5}\left(Q^{*}\right)^{2}
$$

Os cálculos decorrentes das Eq.(3) a Eq.(13) possibilitam desenvolver a Tabela (1), com o uso de dados contidos no catálogo de bomba Centrilift Série 400 , dezoito estágios, freqüência de $60 \mathrm{hz}$ e propriedade do fluido: Lubrax Hydra XP 10.

Tabela 1. Correção de viscosidade do fluido.

\begin{tabular}{|c|c|c|c|c|}
\hline & $\begin{array}{c}\text { Vazão } \\
\left(\mathrm{m}^{\wedge} 3 / \text { dia }\right)\end{array}$ & $\begin{array}{c}\text { Nível } \\
(\mathrm{m})\end{array}$ & $\begin{array}{c}\text { BHPvisc } \\
(\mathrm{HP})\end{array}$ & $\begin{array}{c}\text { Eficiência } \\
(\%)\end{array}$ \\
\hline 0,6 Qbep & 39.02 & 158.1 & 3.6177 & 21.60 \\
\hline 0,8Qbep & 52.03 & 145.6 & 3.9083 & 24.46 \\
\hline Qbep & 67.76 & 126.1 & 4.0423 & 25.26 \\
\hline 1,2Qbep & 78.04 & 98.77 & 4.0138 & 23.63 \\
\hline
\end{tabular}

A potência elétrica denominada BHP (Brake Horse Power) no caso do óleo, $B H P_{\text {óleo }}$, e no caso da água, $B H P_{\text {água }}$, podem ser determinada por meio da Eq.(14), onde $\rho$ é densidade relativa do fluido.

$$
B H P=1,519 * 10^{-4} \frac{Q x H x \rho}{\eta}
$$

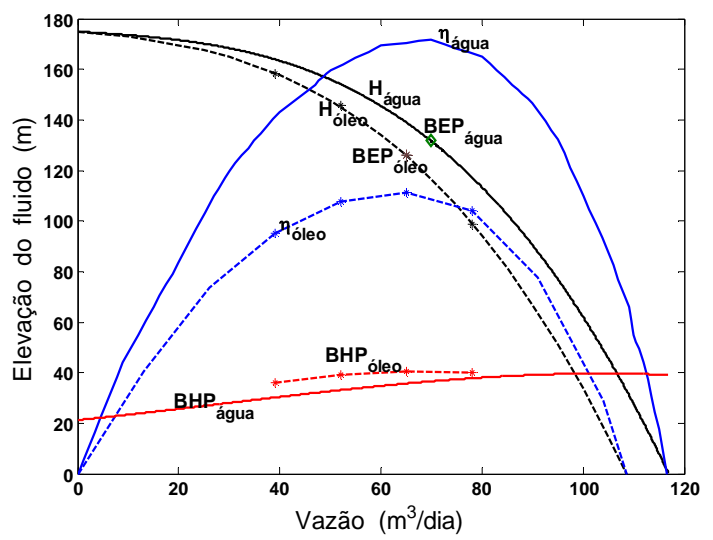

Figura 2. Curva característica com efeito da viscosidade.

$\mathrm{Na}$ Fig.(2), os pontos: 0,6Qbep; 0,8Qbep; 1,0Qbep e 1,2Qbep são mostrados no sentido crescente do eixo vazão volumétrica, respectivamente. Devido à diferença de viscosidade entre os fluidos verifica-se a distinção nos pontos: $\mathrm{BEP}_{\text {água }}$ e $\mathrm{BEP}_{\text {óleo }}$. Também, observa-se uma redução na eficiência e elevação do fluido. Já na potência elétrica observa-se um aumentou de carga.

\section{Não linearidade da planta BCS e controle}

\subsection{Modelo dinâmico do nível de fluido no anular}

O nível dinâmico, $h_{d}$, no anular do poço na Fig.(3), representa a referência para elaboração da estratégia de controle proposta neste artigo. A capacidade volumétrica e pressão de fluido são específicos para cada ponto de rotação da bomba. Onde $h_{e}$ é o nível estático do fluido, na condição de desligamento da bomba. $\mathrm{O}$ diâmetro interno do anular é $d i_{A N}$, e o diâmetro externo da bomba é de $e_{\text {bomba }}$. A planta BCS do LEA possui uma estrutura em que o óleo produzido 
(bombeado pelo método de elevação) é re-injetado no reservatório.

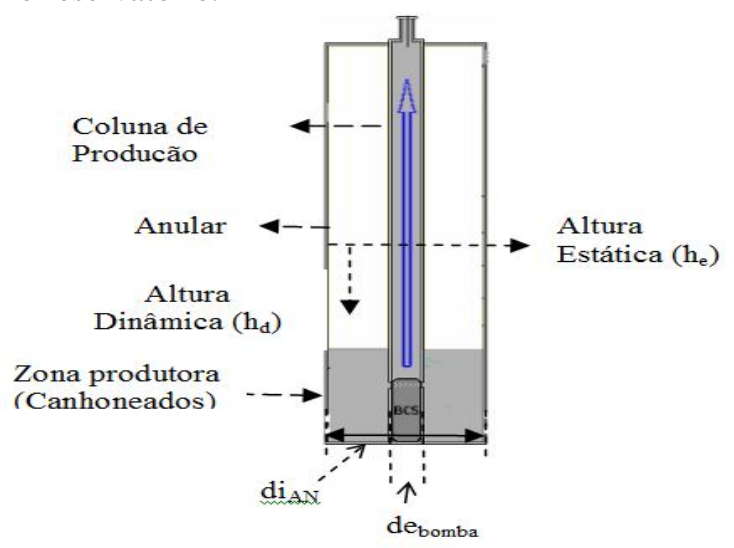

Figura 3: Sistema de bombeio centrifugo submerso.

O fluxo de óleo que escorre na zona produtora, vazão de entrada no anular é denominado $Q_{r}$, já definido na seção 2.1, e vazão de saída do anular $Q_{b}$ (fluxo bombeado). Logo, o balanço volumétrico de líquido no anular do poço, descrito por uma equação diferencial ordinária na Eq.(15).

$$
A_{A N} \frac{d h_{d}}{d t}=Q_{r}-Q_{b}
$$

Em que: $A_{A N}$ é a área da seção transversal do anular do poço. $A_{A N}$ é calculado da seguinte forma: $A_{A N}=\frac{\pi\left(d i_{A N}^{2}-d e_{b o m b a}^{2}\right)}{4}$. E $\frac{d h_{d}}{d t}$ é a taxa de variação do nível $h_{d}(t)$ no anular do poço.

O nível de fluido no anular possui o comportamento não linear mostrado na Fig.(4).

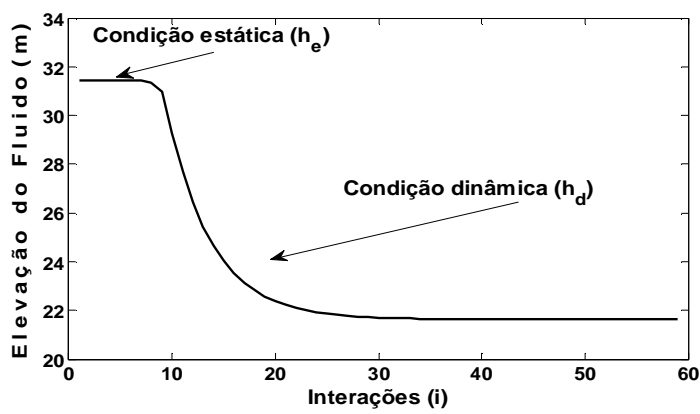

Figura 4. Comportamento do nível de fluido no anular.

Ainda na Fig.(4), pode-se observar que o nível de fluido no anular decresce até um dos pontos de equilíbrio dinâmico, em que a vazão de entrada se estabiliza com a vazão de saída, após a bomba BCS ser ligada. A curva não-linear representa o funcionamento do processo em malha aberta com freqüência específica de $50 \mathrm{~Hz}$.

A Fig.(5) foi desenvolvida, devido a aplicação de leis de afinidades na curva característica com efeito da viscosidade já descrita anteriormente. Esta figura mostra o desempenho esperado do sistema de bombeio para diferentes freqüências de operação.

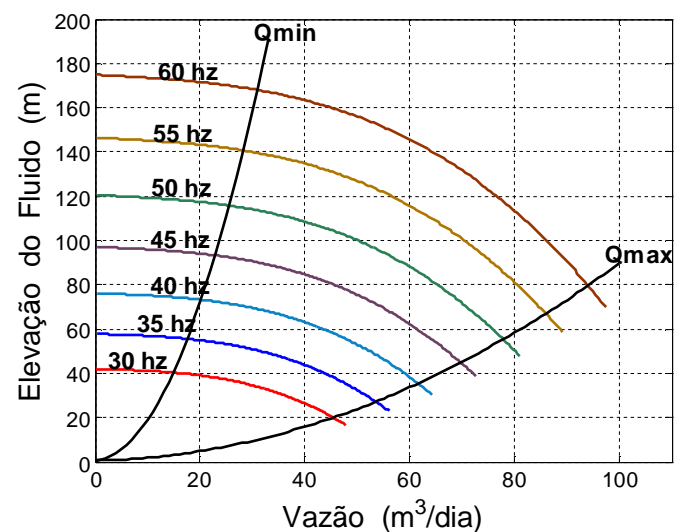

Figura 5. Curva característica elevação do fluido com variação de freqüência.

Contudo, pode-se inferir o perfil não-linear que relaciona a vazão máxima da bomba $Q_{b}$ com sua freqüência de operação, conforme observado na figura acima, por meio da Eq.(16).

$$
\mathrm{Q}_{b}=-1,87 * 10^{-4} \mathrm{f}^{3}+2,31 * 10^{-2} \mathrm{f}^{2}+5,852 * 10^{-1}+9.1945
$$

Onde $f$ é a frequiência de alimentação da bomba em Hertz e $Q_{b}$ vazão da bomba em metros cúbicos por dia. O comportamento da vazão $Q_{b}$ é mostrado na Fig.(6).

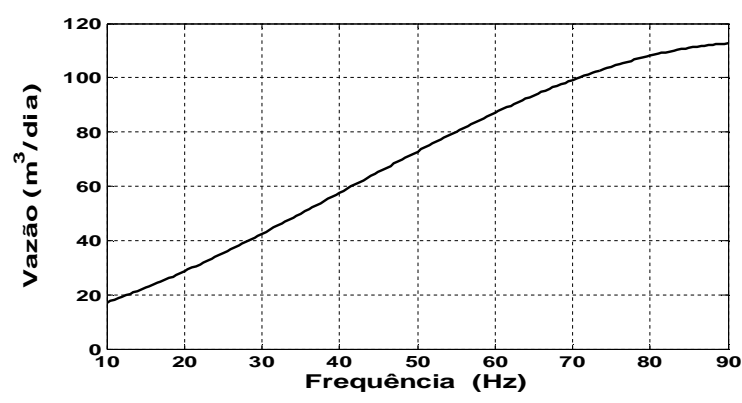

Figura 6. Curva da bomba em função da frequiência.

\subsection{Controle Fuzzy-PID}

O método de controle Fuzzy-PID (Lemke et al., 1985; Melo, 2008) consiste na supervisão de um modelo de controle PID por um controlador inteligente Fuzzy. Este controle propicia um aumento de desempenho devido o controlador não-linear ser capaz de controlar um sistema, também não-linear. Conceitualmente, o controlador inteligente Fuzzy atua na supervisão dos parâmetros PID para controle de processos. Os ajustes dos ganhos PID ocorrem de forma dinâmica, de acordo com o status do processo monitorado. O diagrama de bloco na Fig.(7) representa o controlador com o sistema BCS.

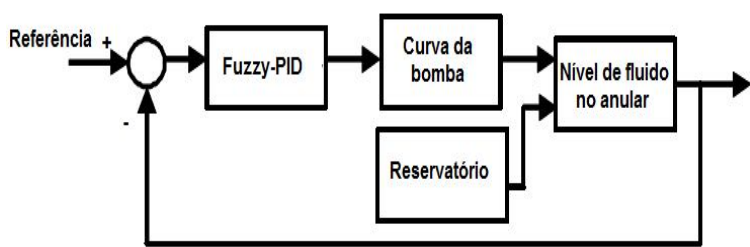

Figura 7. Diagrama de bloco Fuzzy-PID do sistema BCS. 
A estrutura do controle Fuzzy-PID é mostrada na Fig.(8). Onde pode ser observado o erro, $e$, a derivada do erro, $c e$, e a integral do erro ie. Também é possível observar a adaptação dos ganhos a partir do supervisor Fuzzy: proporcional $\Delta K_{p}$, derivativo $\Delta K_{d}$, e integrativo $\Delta K_{i}$. O sinal de controle $u$, o nível de óleo no anular $h_{d}$, e a referência $r$.

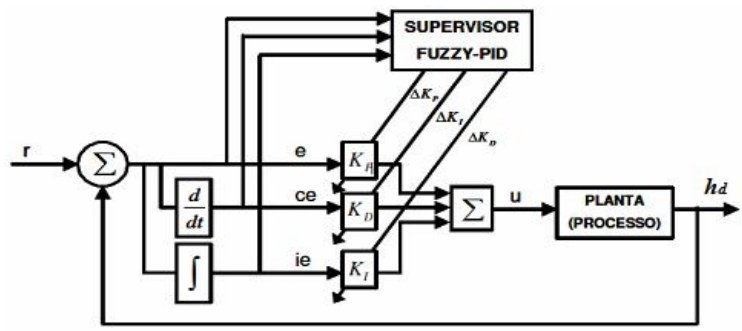

Figura 8. Estrutura do controle Fuzzy-PID.

A Tabela (2) apresenta os conjuntos nebulosos relacionados à base de regras com as variáveis de saída: proporcional $P_{f}$, derivativa $\mathrm{D}_{\mathrm{f}}$, integrativa $I_{f}$

Tabela 2. Conjuntos nebulosos com base de regras.

\begin{tabular}{|c|l|c|c|}
\hline Significado & \multicolumn{1}{|c|}{ Sigla } & $\mathrm{P}_{\mathrm{f}}$ & $\mathrm{D}_{\mathrm{f}}, \mathrm{I}_{\mathrm{f}}$ \\
\hline Muito negativo & $\mathrm{N}--$ & 1,0 & 1,0 \\
\hline Negativo médio & $\mathrm{N}-$ & 2,0 & - \\
\hline Pouco negativo & $\mathrm{N}$ & 3,0 & 2,0 \\
\hline Zero & $\mathrm{Z}$ & 4,0 & 3,0 \\
\hline Pouco positivo & $\mathrm{P}$ & 5,0 & 4,0 \\
\hline Positivo Médio & $\mathrm{P}+$ & 6,0 & - \\
\hline Muito Positivo & $\mathrm{P}++$ & 7,0 & 5,0 \\
\hline
\end{tabular}

Tabela 3. Base de regras da saída $P_{f}$

\begin{tabular}{|c|c|c|c|c|c|c|}
\hline \multicolumn{2}{|c|}{$\mathrm{P}_{\mathrm{f}}$} & \multicolumn{5}{|c|}{ Derivada do erro } \\
\hline \multirow{4}{*}{ Erro } & & $\mathrm{N}--$ & $\mathrm{N}-$ & $\mathrm{Z}$ & $\mathrm{P}$ & $\mathrm{P}++$ \\
\cline { 2 - 7 } & $\mathrm{N}--$ & 1,0 & 1,0 & 1,0 & 1,0 & 1,0 \\
\cline { 2 - 7 } & $\mathrm{N}$ & 1,0 & 1,0 & 1,0 & 1,0 & 1,0 \\
\cline { 2 - 7 } & $\mathrm{Z}$ & 4,0 & 4,0 & 4,0 & 4,0 & 4,0 \\
\cline { 2 - 7 } & $\mathrm{P}$ & 4,0 & 5,0 & 6,2 & 5,0 & 4,0 \\
\cline { 2 - 7 } & $\mathrm{P}++$ & 4,0 & 4,0 & 4,0 & 4,0 & 4,0 \\
\hline
\end{tabular}

Tabela 4. Base de regras das saídas $\mathrm{D}_{\mathrm{f}}, I_{f}$.

\begin{tabular}{|c|c|c|c|c|c|c|}
\hline \multicolumn{2}{|c|}{$\mathrm{D}_{\mathrm{f}}, \mathrm{I}_{\mathrm{f}}$} & \multicolumn{5}{|c|}{ de, ie } \\
\hline \multirow{4}{*}{ Erro } & & $\mathrm{N}--$ & $\mathrm{N}-$ & $\mathrm{Z}$ & $\mathrm{P}$ & $\mathrm{P}++$ \\
\cline { 2 - 7 } & $\mathrm{N}--$ & 1,0 & 1,0 & 1,0 & 1,0 & 1,0 \\
\cline { 2 - 7 } & $\mathrm{N}$ & 1,0 & 1,0 & 1,0 & 1,0 & 1,0 \\
\cline { 2 - 7 } & $\mathrm{Z}$ & 1,0 & 1,0 & 5,0 & 4,0 & 3,0 \\
\cline { 2 - 7 } & $\mathrm{P}$ & 1,0 & 1,0 & 4,0 & 3,0 & 3,0 \\
\cline { 2 - 7 } & $\mathrm{P}++$ & 1,0 & 1,0 & 3,0 & 3,0 & 3,0 \\
\hline
\end{tabular}

A Tabela (3) mostra a base de regras da saída $P_{f}$ enquanto que a Tabela (4) mostra as regras das saídas $D_{f}$ e $I_{f}$. Já a estrutura das funções de pertinências utilizadas neste artigo é mostrada conforme descrito: a Fig.(9) relaciona as entradas $e, c e$, ie com o grau de pertinência; a Fig.(10) saída $P_{f}$, e Fig.(11) as saídas $D_{f}, I_{f}$ com os respectivos graus de pertinências.

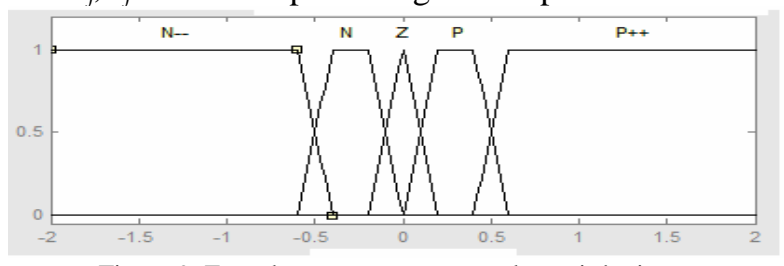

Figura 9. Entradas $e$, $c e$, ie com grau de pertinência.

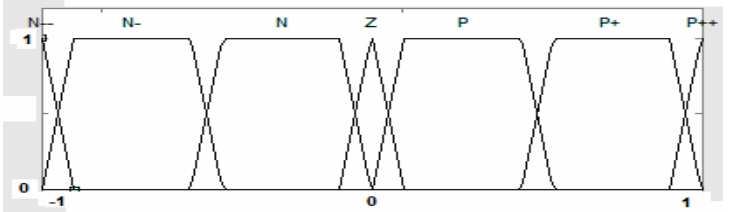

Figura 10. Saída $P f$ com grau de pertinência.

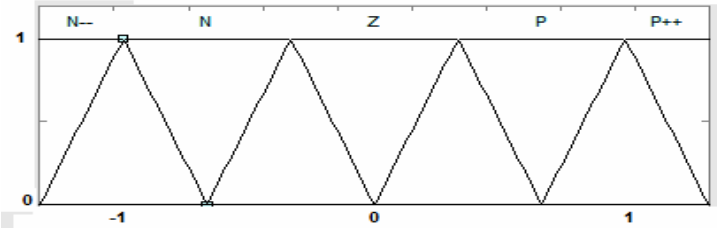

Figura 11. Saídas $D f$ e If com grau de pertinência

\section{Resultados}

O controle Fuzzy-PID proposto neste artigo para controlar o nível de fluido no anular do poço foi simulado com base na Fig.(8) no Simulink. Alguns parâmetros adquiridos na planta experimental de bombeio em malha aberta foram utilizados aqui. Por exemplo, $h_{e}=31,44 \mathrm{~m}, A_{A N}=0,016 \mathrm{~m}^{2}, \gamma=8388 \mathrm{Nm}^{-3}$, $P_{r}=263,4 K P a, \quad I P R=0,886 \mathrm{~m}^{3} / \mathrm{dia} / \mathrm{KPa}$ e faixa de freqüência entre $30 \mathrm{~Hz}$ a $60 \mathrm{~Hz}$. A Fig.(12) apresenta o comportamento do nível de fluido, $h_{d}$. A Fig.(13) mostra o sinal de controle Fuzzy-PID, $u$, que alimenta a bomba BCS. Já a Fig.(14) representa um comparativo entre a vazão de entrada $Q_{r}$ e a vazão de saída $Q_{b}$.

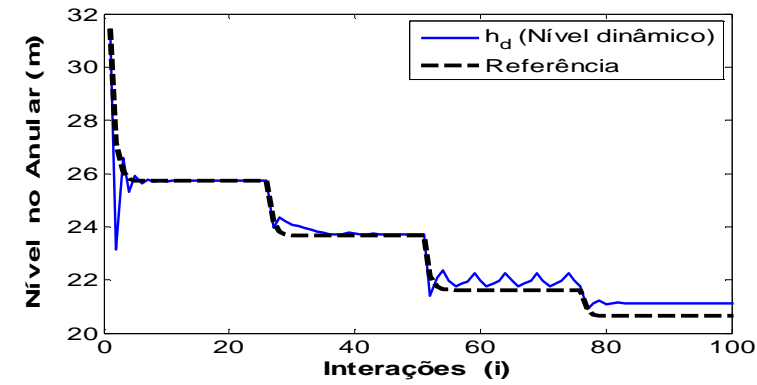

Figura 12. Nível de fluido no alunar, devido ação de controle.

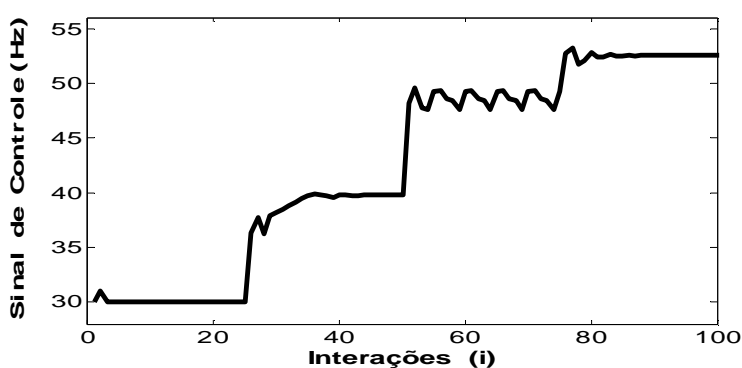

Figura 13. Sinal de controle Fuzzy-PID que alimenta a bomba.

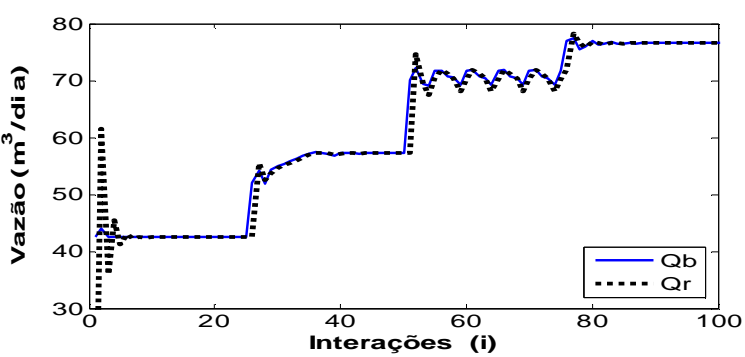

Figura 14. Vazão de entrada $Q r$ e vazão de saída $Q b$ no anular. 
Conforme observado na Fig.(12), o nível de fluido no anular responde com estabilidade e seguindo o sinal de referência em relação ao sinal de controle Fuzzy-PID Fig(13), que atua na bomba BCS. Assim sendo, quando a bomba é acionada, o nível de óleo inicialmente na condição estática, $h_{e}=31,44 m$, passa a descrever novos pontos de operação dinâmica, $h_{d}$, em formato degrau decrescente. Neste sentindo, a Fig.(14) representa um comparativo entre a vazão de entrada $Q_{r}$ e a vazão de saída $Q_{b}$, que representam alguns pontos de produção da planta aqui em análise. Estes pontos de equilíbrio dinâmico ocorrem quando a vazão de entrada do reservatório se iguala com a vazão da bomba.

A Fig.(15) apresenta os ganhos $K_{p}, K_{d}$ e $K_{i}$ ajustados pelo supervisor Fuzzy. Já a Fig.(16), Fig(17) e Fig.(18) representam a componente do sinal de atuação proporcional $U_{p}$, integral $U_{i}$ e derivativo $U_{d}$, respectivamente.

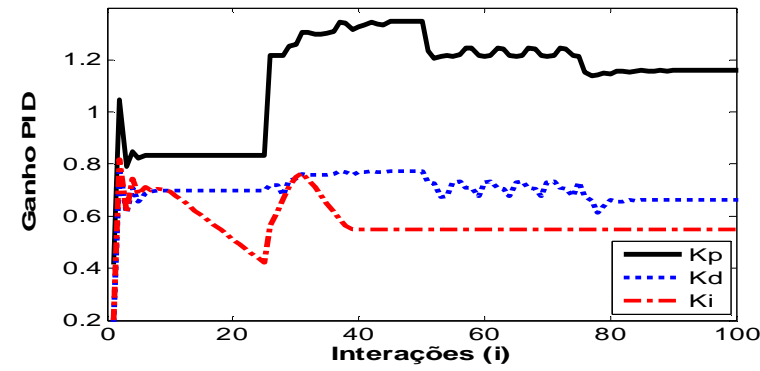

Figura 15. Ajuste dos ganhos $K p, K d$ e $K i$.

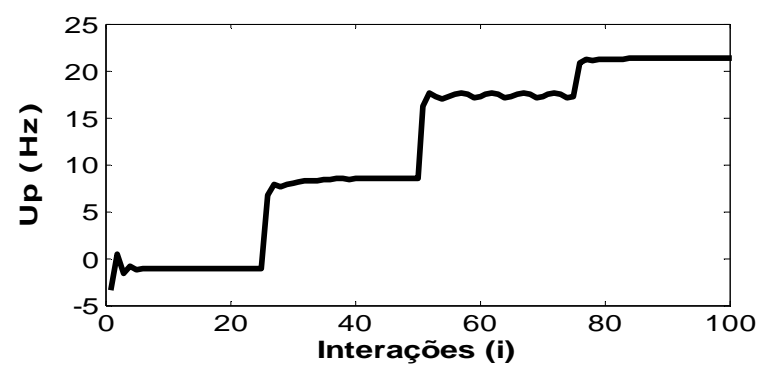

Figura 16. Sinal de controle Proporcional.

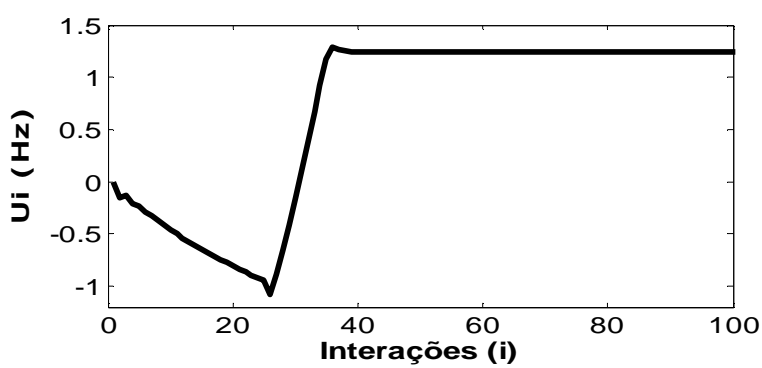

Figura 17. Sinal de controle Integral.

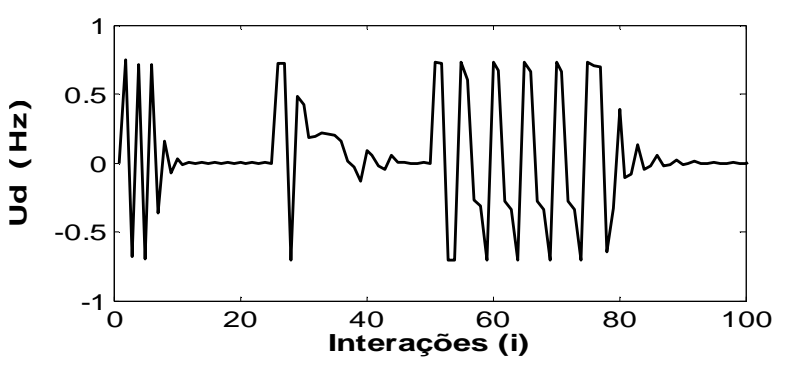

Figura 18. Sinal de controle derivativo.

\section{Conclusão}

O presente trabalho utilizou técnica de controle Fuzzy-PID para controlar o nível de óleo no anular de uma planta de bombeio centrifugo submerso. Verificou-se em ambiente simulado que o sistema BCS se manteve estável mesmo na presença de variáveis não modeladas e incertezas intrínsecas ao processo. Por fim, devido ao aumento do sinal de freqüência que alimenta a bomba, ocorre proporcionalmente um aumento na vazão de saída do anular que diminui o seu nível de óleo, que conseqüentemente passa a operar em novo ponto de equilíbrio. Isto ocorre, em virtude da capacidade do reservatório, em suprir a depleção (perda de pressão) do nível no anular com vazão de entrada no poço nos canhoneados. Na sequência deste trabalho o controlador proposto será implementado no sistema físico de bombeio centrifugo submerso do LEA.

\section{Agradecimentos}

Os autores agradecem ao CTAI da Escola Politécnica da UFBA pela infra-estrutura disponibilizada, a CAPES pelo apoio financeiro e a Petrobrás pelo apoio ao desenvolvimento do LEA.

\section{Referências Bibliográficas}

Amaral, G. D. L. (2007). Modelagem do Escoamento Monofásico em Bomba Centrífuga Submersa Operando com Fluidos Viscosos. Universidade Estadual de Campinas. 234p.

Estevam, V. 2002. Uma Análise Fenomenológica da Operação de Bomba Centrífuga com Escoamento Bifásico. Universidade Estadual de Campinas, 265p.

Estevam, V. 2008. Curso Avançado de BCS. Apostila Petrobrás. $332 \mathrm{p}$.

Lemke, V. N., H. R., De-Zhao, W., 1985. Fuzzy PID supervisor. Proceedings of the 24th IEEE Conference on Decision and Control, Fort Lauderdale, Florida, USA. 602 - 608p.

Maitelli, C. W. S. P., 2010. Simulação do Escoamento Monofásico em um Estágio de uma Bomba Centrifuga Utilizando Técnicas de Fluidodinâmica Computacional. Universidade Federal do Rio Grande do Norte. 183p.

Melo, A. V., 2008. Desenvolvimento de Controladores Inteligentes para o Sistema VASPS. Universidade Estadual de Campinas. $134 \mathrm{p}$.

Ribeiro, M.P., Oliveira, P.S., Matos, J.S., Silva, J.E.M. Field Applications of Subsea Electrical Submersible Pump in Brasil. Offshore Technology Conference, OTC 17415, Houston, Texas, May 2005.

Rossi, N. C. M., 2008. Bombeio Centrifugo Submerso. Apostila Petrobrás. $70 \mathrm{p}$.

Takács, G., 2009. Electrical submersible pumps manual. Gulf Professional Publishing, USA.

Turzo, Z., Takács, G. and Zsuga, J., 2000. A Computerized Model for Viscosity Correction of Centrifugal Pump Performance Curves. $47^{\text {th }}$ Southwestern Petroleum Short Course, Texas.

Verde, W. M. 2011, Estudo Experimental de Bombas de BCS Operando com Escoamento Bifásico Gás-Líquido. Universidade Estadual de Campinas. 129p. 